\title{
Low Energy Adaptive Clustering Hierarchy based routing Protocols Comparison for Wireless Sensor Networks
}

\author{
C. Rajivegandhi \\ Department of Computer Science \& Engineering, SRM University, \\ rajivegandhi@gmail.com \\ K. Raghavi \\ kumaravelsrm@gmail.com
}

\author{
K.S. Jeyshree \\ Chennai, India \\ arthimohan09@gmail.com
}

\begin{abstract}
As A Result Of Recent Advances In Microelectronic System Fabrication, Progress In Ad-Hoc Networking Routing Protocols, Integrated Circuit Technologies, Wireless Communications, Microprocessor Hardware And Nano-Technology, Pervasive Computing, Embedded Systems And Distributed Signal Processing, Wireless Sensor Networks (Wsns) Have Emerged In The Past Decade. Recent Advances In Wireless Sensor Networks Have Led To Many New Protocols Specifically Designed For Routing, As Routing Protocols Are Application Specific. Efficient Routing In A Sensor Network Requires That The Routing Protocol Must Minimize Energy Dissipation And Maximize Network Life Time. Hierarchical Routing (Cluster Based) Protocols Are Well-Known Techniques With Special Advantages Related To Energy Efficiency, Scalability And Efficient Communication. LEACH (Low Energy Adaptive Clustering Hierarchy) Is The First Hierarchical Routing Protocol In WSN. In This Paper We Compare Different Hierarchical Routing Protocols That Are Derived With The Basic Principle Of LEACH. Various Parameters Like Number Of Hops, Energy Consumption, Latency Location Information Etc. Are Used For Comparison. Comparison Results Clearly Show That InterIntra Cluster Multihop-LEACH Performs Better Than Other Types Of LEACH Protocols.
\end{abstract}

Keywords: Wsn, Clustering, Leach, E-Leach, C-Leach,V-Leach, Tr-Leach, M-Leach, Inter-Intra Cluster Multihop: $L E A C H$

\section{Introdauction}

Wireless sensor network (WSN) [1,2] consists of hundreds and even thousands of small tiny devices called sensor nodes (motes) distributed autonomously to monitor physical or environmental conditions, such as temperature, vibration, sound ,motion and pressure at different locations. Motes usually consist of a battery, a small amount of memory, low clock rate processor and a component to allow wireless communication. Motes also have sensors attached to them to monitor the physical environment in some way. Energy plays an important role in wireless sensor network, and preserving energy of each node is an important goal that must be considered when developing a routing protocol for wireless sensor networks $[3,4]$.

Hierarchical routing protocols are well-known techniques with special advantages related to energy efficiency, scalability and efficient communication. LEACH (Low-Energy Adaptive Clustering Hierarchy) is the first hierarchical routing protocol in WSN. This paper will look at different routing protocols that are derived with the basic principles of LEACH protocol to assess their suitability for use in wireless sensor networks and also compare them. Section 2 contains classification of routing protocols, section 3 contains description of hierarchical routing protocols, Section 4 contains comparison different versions of LEACH protocols. Finally section 5 contains conclusion and future work.

\section{Classification Of Network Protocols}

According to the network structure and protocol peration[5] almost all the routing protocols can be classified as shown in figure 1 .

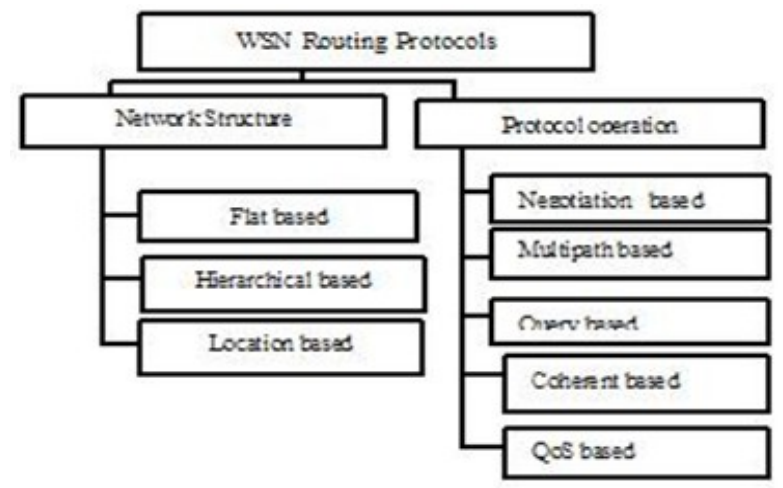

Fig. 1: Classification of WSN Routing Protocols 


\subsection{Network Structure}

Based on structural orientation of base stations and the structural orientation of sensor nodes we classify routing protocols as flat based, hierarchical based and location based.

Flat based: In these networks, all nodes play the same role and there is absolutely no hierarchy. To any reachable sensor node within the sensor cloud flat routing protocols distribute information as needed. No effort is made to organize the network or its traffic to discover the best route hop by hop to a destination by any path.

Hierarchical based: This class of routing protocols sets out to attempt to conserve energy by arranging the nodes into clusters as shown in Figure 2. Nodes in a cluster transmit to a head node within close proximity which aggregates the collected information and forward this it to the base station [6]. Good clustering protocols play an important role in network scalability as well as energy efficient communication. Clusters may lead to a bottleneck, on the negative side of it.

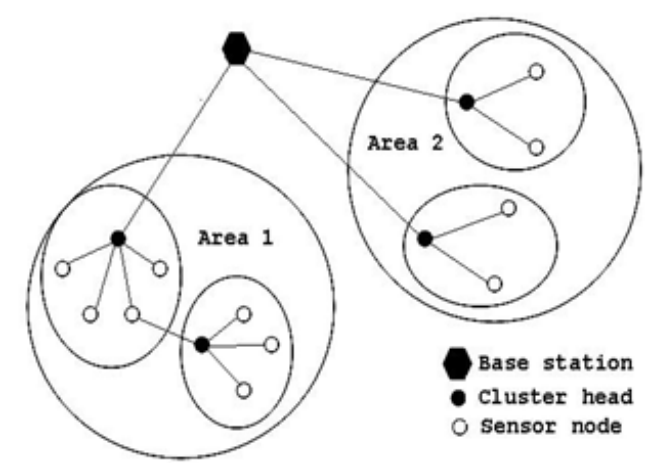

Fig. 2: Clustering Nodes

Location based: Most of the routing protocols for sensor networks require location information for sensor nodes. To calculate the distance between two particular nodes, in most cases location information is needed so that energy consumption can be estimated. Location information can be utilized in routing data in an energy efficient way, since there is no addressing scheme for sensor networks like IP-addresses.

\subsection{Protocol Operation}

The main operating characteristic of a routing protocol is described by it; in terms of communication pattern, hierarchy, delivery method, computation, next- hop.

Multipath based: In this case, the network derives benefit from the fact that there may be multiple paths between a source node and the destination. Different paths usage ensures that energy is depleted uniformly and no single node bears the brunt [3].

Query based: Here the focus lies on propagation of queries throughout the network by the nodes which require few data. Any node which has the requested data and receives a query, replies with the data to the requesting node. This approach conserves energy by minimizing redundant or non-requested data transmissions [7].

Negotiation based: The nodes here exchange a number of messages between themselves before transmission of data. The benefit of this is that redundant data transmissions are suppressed. It should however be ensured that the transmissions negotiation are not allowed to exceed an extent that the energy saving benefit is offset by the negotiation overhead.

QoS-based: QoS based protocols have to find a trade-off between energy consumption and the quality of service. A high energy consumption path or approach may be adopted if it improves the QoS. So when interested in energy conservation, these types of protocols are usually not very useful.

Coherent-based : Coherence based protocols focus on how much data processing takes place at each node. In coherent protocols, data is sent to an aggregator node after minimum possible processing, and processing is then done at the aggregator. However, the aggregator nodes must have more energy than the other ordinary nodes, or else they will be depleted rapidly.

\section{Discription Of Hierarchical Routing Protocols}

In a hierarchical architecture[5,6], higher energy nodes can be used to process and send the information while low energy nodes can be used to perform sensing in the proximity of the target. This means that assigning special tasks to cluster heads and creation of clusters can greatly contribute to overall system lifetime, scalability and energy efficiency. An efficient way to lower energy consumption within a cluster and perform data aggregation and fusion in order to decrease the number of transmitted messages to the BS is hierarchical routing. 


\subsection{Low-Energy Adaptive Clustering Hierarchy (LEACH)}

LEACH is a first hierarchical routing protocol proposed by Wendi B. Heinzelman, et al [6]. In LEACH, nodes organize themselves into clusters and all non-cluster head nodes transmit to the cluster-head.

The cluster head performs aggregation of data and transmits the data directly to the base station as shown in figure 3. These self elected cluster heads continue to be cluster heads for a period referred to as a round. The operation of LEACH is separated into two phases: the setup phase and the steady state data transfer phase.

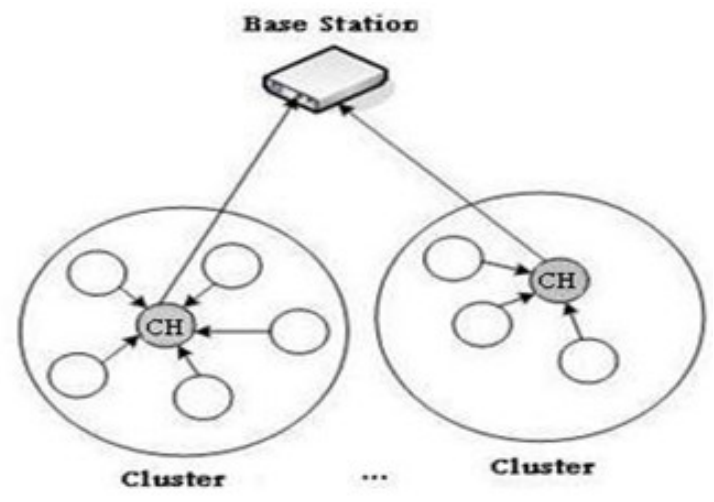

Fig3: Each Cluster Head Transmit the Data Directly to the Base Station

During the setup phase, cluster heads are selected based on the random number chosen for the network and the number of times the node has been a cluster-head so far. This decision is made by each node $\mathrm{n}$ choosing a random number between 0 and 1 . The node, becomes a cluster-head for the current round, if the number is less than a threshold $\mathrm{T}(\mathrm{n})$. The threshold is formed as follows:

$$
T(n)= \begin{cases}\frac{P}{1-P\left(r \bmod \frac{1}{P}\right)} & \text { if } n \in G(1) \\ 0 & \text { otherwise }\end{cases}
$$

where the desired cluster-head probability is $\mathrm{P}, \mathrm{r}$ is the number of the current round and $\mathrm{G}$ is the set of nodes that have not been cluster-heads in the last $1 / \mathrm{P}$ rounds.

Once the nodes have elected themselves to be cluster heads, they sets up a TDMA schedule and transmits the schedule to all the nodes in its cluster, completing this setup phase, which is then followed by a steady-state operation. This steady state operation is broken into frames, where nodes send their respective data to the cluster head at most once per frame during their allocated slot.

\subsection{Energy-LEACH (E-LEACH)}

Fan el. Al. proposes a new protocol known as Energy-LEACH in which $\mathrm{CH}$ selection procedure is improved. It makes residual energy of node as the main metric which decides whether the nodes turn into $\mathrm{CH}$ or not after the first round. Like LEACH protocol, E-LEACH protocol has been divided into rounds. Every node has the same probability to turn into $\mathrm{CH}$ that means nodes are randomly selected as $\mathrm{CHs}$ which is the first round. I the residual energy of each node is different after one round communication and taken into account for the selection of the $\mathrm{CHs}$ is the next round. That mean nodes that have more energy will become a CHs rather than nodes with less energy.

E-LEACH tries to optimize the energy consumption of the network by ensuring that nodes belonging to hot regions have a high probability of becoming a cluster heads. Thus nodes belonging to hot regions, which are expected to transmit data more frequently, now do it over shorter distances, thereby leading to balanced energy consumption over the network. E-LEACH selects a node to be a cluster head depending upon its hotness value and residual energy. This is an improvement over stochastic approach used in LEACH in terms of energy efficiency.

\subsection{Centralized-LEACH (C-LEACH)}

Wendi et al. [9] proposed LEACH-C protocol which uses a centralized algorithm. LEACH offers no guarantee about the placement and/or number of cluster heads. In [9], an enhancement over the LEACH protocol was proposed. The protocol, called LEACH-C, uses an algorithm of centralized clustering and the same 
steady-state phase as LEACH. LEACH-C protocol can produce better performance by dispersing the cluster heads throughout the network. During the set-up phase of LEACH-C, the current location (possibly determined using GPS) information is send by each node and residual energy level to the sink. The steady-state phase of $\mathrm{LEACH}-\mathrm{C}$ is similar to that of the LEACH protocol.

\subsection{Vice cluster head-LEACH (V-LEACH)}

In new version of $\mathrm{LEACH}$ [10] protocol, the cluster contains; $\mathrm{CH}$ (responsible only for sending data that is received from the cluster members to the $\mathrm{BS}$ ), vice-CH (the node that will become a $\mathrm{CH}$ of the cluster in case of $\mathrm{CH}$ dies), cluster nodes (gathering data from environment and send it to the $\mathrm{CH}$ ) as shown in figure 4 . In the original leach, the $\mathrm{CH}$ is always on receives data from cluster members, assemble these data and then send it to the BS which might be located far away from it.

Than the other nodes $\mathrm{CH}$ will die earlier in the cluster because of its operation of receiving, sending and overhearing. When the $\mathrm{CH}$ die, as the data is gathered by cluster nodes which will reach the base station the cluster will become useless. In our V-LEACH protocol, besides having a $\mathrm{CH}$ in the cluster, there is a vice- $\mathrm{CH}$ that takes the role of the $\mathrm{CH}$ when the $\mathrm{CH}$ dies because the reasons we mentioned above. By doing this, cluster nodes data will always reach the BS; there is no need to elect a new $\mathrm{CH}$ each time the $\mathrm{CH}$ dies. As this will extend overall network life time.

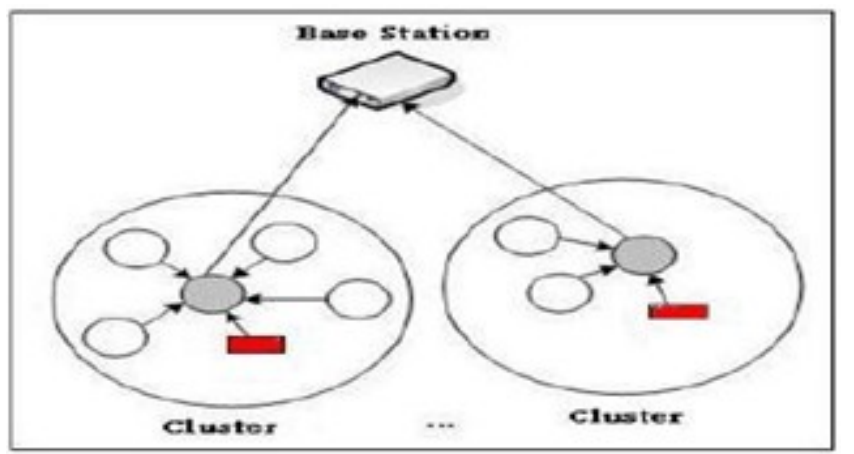

Fig 4: V-LEACH containing both $\mathrm{CH}$ and Vice $\mathrm{CH}$ (in red colour)

\subsection{Two - level LEACH (TL- LEACH)}

Two-Level Hierarchy LEACH [11] is a proposed extension to the LEACH algorithm. In addition to the other simple sensing nodes it utilizes two levels of cluster heads (primary and secondary). According to this algorithm, the primary cluster head communicates with the secondary, and the corresponding secondary communicate with the nodes in sub-cluster. Data-fusion can also be performed as in LEACH. In addition, communication within a cluster is still scheduled using TDMA time-slots. The two-level structure of TLLEACH to be transmitted to base station reduces the amount of nodes, which effectively reduces the total energy usage.

\subsection{Multihop-LEACH (M-LEACH)}

M-LEACH is the modified version of LEACH [10]. M-LEACH protocol operates similar to LEACH protocol, but changes communication mode from single hop to multi-hop between CHs (Cluster Heads) and BS (Base Station). Multihop-LEACH protocol selects an optimal path between all CHs and the BS, then, according to the selected optimal path, data is to the BS as shown in figure 5.

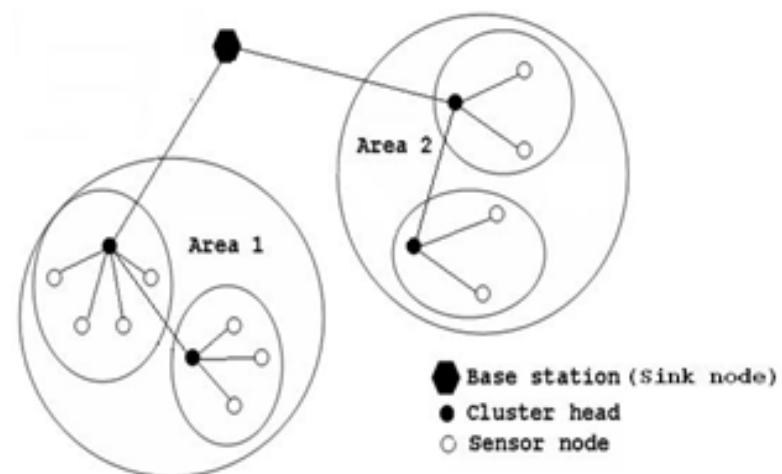

Fig 5: Nodes communicate to Base Station through an optimal path of Cluster Heads 


\subsection{Inter-Intra Cluster Multihop-LEACH}

There are two major modifications in Inter-intra luster Multihop-LEACH protocol with respect to MLEACH protocol [12] as shown in figure 6.

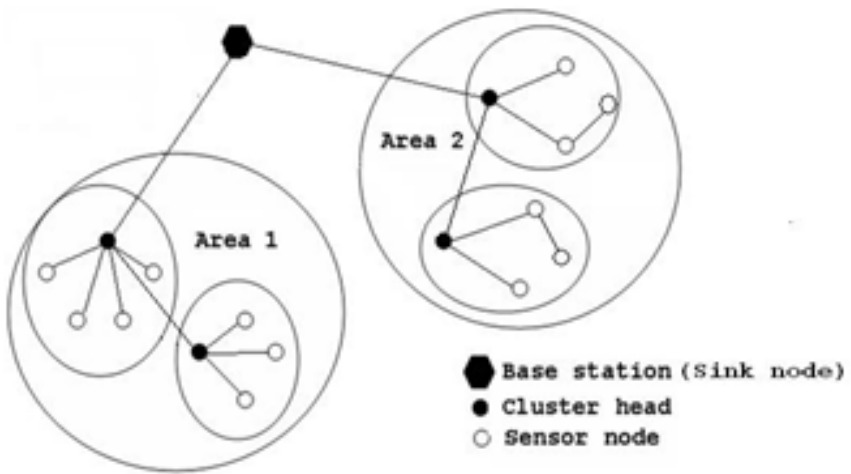

Fig 6: Nodes Communicate to Base Station using inter and intra cluster multihop operation

Inter-cluster multihop operation - In this model network is grouped into different clusters. Each cluster is composed of one cluster head $(\mathrm{CH})$ and cluster member nodes. The respective $\mathrm{CH}$ from its cluster member nodes gets the sensed data, assembles the sensed information and later sends it to the Base Station through an optimal multihop tree formed between cluster heads (CHs) with base station as root node as shown in figure 6 .

Intra-cluster Multihop operation - However, we note that in general using single hop communication within a cluster for communication between the sensor nodes and the cluster heads may not be the optimum choice. When the sensor nodes are deployed in regions of dense vegetation or uneven terrain, it may be beneficial to use multihop communication among the nodes in the cluster to reach the cluster head. As it is possible for nodes to remain disconnected from the network due to a cluster head not being in range, each node is able to request that another connected node to become a cluster head.

\section{Comparison Of Versions Leach Based Protocols}

The parameters used in comparing the various LEACH based protocols are Number of hops used in communicating, Residual energy of nodes used for selecting CH, Energy consumption, Latency, Loss of data, Location information and Overall Network life time shown in table 1.

\section{Conclusion And Future Work}

In this paper we considered a well known protocol for WSN called LEACH which is the first and the most important protocol in wireless sensor network which uses cluster based broadcasting technique. We narrated different protocols that are derived with the basic principle of LEACH protocol. Then we compared various protocols and this comparison led us to the conclusion that Inter-Intra Cluster Multihop-LEACH performs better than all the LEACH based protocols that we considered.

Future work could include improving the protocols developed during this report and the development of new clustering techniques to minimize the energy consumption.

\begin{tabular}{|l|l|l|l|l|l|l|l|} 
Protocols & $\begin{array}{l}\text { Number of hops used } \\
\text { in communicating }\end{array}$ & $\begin{array}{l}\text { Residual energy of } \\
\text { hodes used for } \\
\text { selecting CH }\end{array}$ & $\begin{array}{l}\text { Energy } \\
\text { consumption }\end{array}$ & Latency & $\begin{array}{l}\text { Loss } \\
\text { of data }\end{array}$ & $\begin{array}{l}\text { Location } \\
\text { information used } \\
\text { to select CH }\end{array}$ & $\begin{array}{l}\text { Network life } \\
\text { time }\end{array}$ \\
\hline LEACH & Single & No & Medium & Low & More & No \\
\hline E-LEACH & Single & Yes & Less & Low & More & No & Bverage \\
\hline C-LEACH & Single & Yes & Less & Low & Less & Yes & Better \\
\hline V-LEACH & Single & Yes & Less & Low & Less & No \\
\hline TL-LEACH & Two & Yes & Less & More & Less & No & Good \\
\hline M-LEACH & Multihop & Yes & Less & More & Less & No \\
\hline $\begin{array}{l}\text { Inter-Intra } \\
\text { Cluster } \\
\text { Multihop- } \\
\text { LEACH }\end{array}$ & Multihop & & Least & More & Least & No & Best \\
\hline
\end{tabular}

Table 1: Comparison of different hierarchical LEACH based Protocols 


\section{References}

[1]. Sarjoun S. Doumit, Dharma P. Agrawal, "Self- Organizing and Energy-Efficient Network of Sensors", IEEE, pp. 1-6 (2002).

[2]. I.F. Akyildiz, W Su, Y. Sankarasubramaniam and E Cayirci, "Wireless Sensor Networks, a Survey," Communication Magazine, IEEE, Vol. 40 Issue 8, pp. 102-114, August 2002.

[3]. C. Rahul, J. Rabaey, "Energy Aware Routing for Low Energy Ad Hoc Sensor Networks", IEEE Wireless Communications and Networking Conference (WCNC), vol.1, March 17-21, 2002, Orlando, FL, pp. 350-355.

[4]. K. Akkaya, M. Younis, “A Survey on Routing Protocols for Wireless Sensor Networks,” Vol 3, pp. 325-349, May 2005 .

[5]. Rajashree.V.Biradar, V.C .Patil, Dr. R. R Mudholkar, Dr. S. R. Sawant, "Classification And Comparison Of Routing Protocols In Wireless Sensor Networks",Ubiquitous Computing and Communication Journal volume 4, pp.704-711, 2009.

[6]. W.R. Heinzelman, A. Chandrakasan, and H. Balakrishnan, "Energy-efficient communication protocol for wireless microsensor networks," Proceedings of the 33rd Hawaii International Conference on System Sciences, 2000.

[7]. D. Braginsky and D. Estrin,"Rumor Routing Algorithm For Sensor Networks", International Conference on Distributed Computing Systems (ICDCS'01), November 2001.

[8]. W.Heinzelman, J. Kulik, and H. Balakrishnan, "Adaptive Protocols for Information Dissemination in Wireless Sensor Networks", Proc. $5^{\text {th }} \mathrm{CM} / \mathrm{IEEE}$ Mobicom Conference.

[9]. Wendi B. Heinzelman, Anantha P. Chandrakasan and Hari Balakrishnan (2002) IEEE Transactions on Wireless Communications, Vol. 1, No. 4.

[10]. M. Bani Yassein, A. Al-zou'bi, Y. Khamayseh, W. Mardini, International Journal of Digital Content Technology and its Applications, Vol. 3, No. 2, pp. 132-136,2009.

[11]. Loscri V., Morabito G., Marano S. (2005) Vehicular Technology Confer ence, VTC-2005, Volume: $3,1809-1813$.

[12]. Rajashree.V.Biradar, Dr. S. R. Sawant, Dr. R. R. Mudholkar, Dr. V.C .Patil , "Inter-Intra Cluster Multihop-LEACH Routing In Self- Organizing Wireless Sensor Networks", International Journal of Research and Reviews in Computer Science (IJRRCS) Vol. 2, No. 1, March 2011. 